\title{
Effects of a shift from a mixed diet to a lacto-vegetarian diet on some coronary heart disease risk markers
}

\author{
Gunnar Johansson $^{1^{*}}$, Börje Källgård ${ }^{2}$, Per-Arne Öckerman ${ }^{2}$ \\ ${ }^{1}$ School of Social and Health Sciences, Halmstad University, Halmstad, Sweden; ${ }^{*}$ Corresponding Author: gunnar.johansson@hh.se \\ ${ }^{2}$ Department of Clinical Chemistry, Lund University, Lund, Sweden
}

Received 12 October 2011; revised 15 November 2011; accepted 4 December 2011

\begin{abstract}
Background: There is convincing evidence that vegetarians have lower incidence of coronary heart disease, but there is a debate as to why this is the case. Objective: The aim of the study was to investigate whether a shift from a mixed diet to a lacto-vegetarian diet would lead to a decrease in risk for coronary heart diseases indicated by surrogate markers. Design: Twenty volunteers participated in the study (4 men and 16 women, mean age 44 years, range 27 - 61) from a town in western Sweden. Clinical examinations were performed, blood samples were drawn and dietary survey, i.e. repeated 24-h recalls were carried out before ( 0 month) and 3, 6 and 12 months after the change from a mixed diet to a lacto-vegetarian diet. A dietician educated the volunteers with regard to the vegetarian dietary regimen, organized and taught the vegetarian cooking courses. Results: The dietary shift lead to an increase in the intake of total carbohydrates and fibre and a decrease in fat, protein and sucrose. The coronary heart disease risk markers body weight, body mass index, systolic and diastolic blood pressure, total cholesterol and low-density lipoptrotein cholesterol decreased significantly. Conclusions: There was a decrease in disease risk markers even though the ratio polyunsaturated to saturated fatty acids was unchanged. The main finding in this study is that there was a weight loss, sustained for one year, without any recommendation to decrease the energy intake or any focus on weight reduction.
\end{abstract}

Keywords: Diet; Vegetarian Diet; Coronary Heart Diseases

\section{INTRODUCTION}

There is convincing evidence that vegetarians have lower incidence of coronary heart disease, largely explained by lower low-density lipoprotein (LDL) cholesterol, lower prevalence of obesity and probably a healthier lifestyle [1]. Plant foods contain many beneficial compounds that, acting through multiple mechanisms, provide protection against the disease. The foods mainly mentioned are fruits, vegetables, whole grains, soy, pulses, antioxidant rich foods and foods with a low glycemic index [2]. It may also be important that plant foods usually have a low fat content, especially saturated fatty acids and transfatty acids. A low intake of some foods may also be of importance, such as meat, fast foods, alcoholic and some other beverages, sugary snacks, sweets etc. [3]. Many food components, either by their presence or absence, may thus contribute to the lower disease incidence in vegetarians. Another theory to explain the lower disease incidence is that the various nutrients and phytochemicals are of less importance compared to the overall lower body weights for vegetarians [4-6]. A second reason why this dietary shift is interesting to study is that it previously has been shown that it led to a decrease in colon cancer risk markers $[7,8]$.

The aim of the study was to investigate whether a shift from a mixed diet to a lacto-vegetarian diet would lead to a decreased risk for coronary heart diseases (CHD) indicated by surrogate markers.

\section{SUBJECTS AND METHODS}

\subsection{Subjects}

Twenty volunteers participated in the study (4 men and 16 women, mean age 44 years, range 27 - 61). A call for participants was made by local press and radio and the volunteers were selected after taking part in a clinical examination together with an interview. The selection criteria were that they should have no diseases registered by a general practioner. The selected subjects were healthy, nonsmoking, omnivores and were not taking any prescribed medication. The study was approved by the Ethical Committee of the University of Göteborg, Gothenburg, Sweden and was performed in accordance with the Helsinki declaration. 


\subsection{Study Design}

The volunteers were divided into three groups, each of which started their dietary shift at different time periods of the year, March, May and August. This was done to ensure that any observed alteration would be due to a change from a mixed to a lacto-vegetarian diet rather than to seasonal variation in the diet. The subject participated in a vegetarian cooking course and received lectures in basic nutrition. The courses were held once a week for a period of three months. We defined the starting point of the dietary change as the time when the participants commenced these courses and, thus, started to change their diet. A dietician educated the volunteers with regard to the dietary regimen. The lacto-vegetarian diet introduced by Are Waerland was used as a guide since this diet is recommended by the Swedish Vegetarian Society and therefore most likely the diet that vegetarians in Sweden and Scandinavia eat [9]. This diet is characterized by large amounts of raw vegetables, fruits, unrefined foods and wholemeal products with the exclusion of meat, poultry, fish and eggs. The consumption of soft drinks, coffee, tea, alcoholic beverages, candies, sugar, salt and all refined and instant foods was discouraged. Dairy products were allowed, but fermented rather than unfermented products were recommended.

\subsection{Dietary Survey}

Dietary survey, i.e. repeated 24-h recalls were carried out before ( 0 month) and 3, 6 and 12 months after the dietary change. Four 24-h recalls were performed at each time period, each participant was thus interviewed 16 times. The first 24-h recall for each time period was made in the home of the participant and the following three by telephone. These latter interviews were carried out at unannounced times and at irregular intervals. On all occasions, the interviews covered a Sunday, a Monday, a Tuesday, and a Wednesday. At the first interview the participants received training in estimating the volumes and weights of foods. This was carried out with the aid of models of food items in two or three dimensions and with the volunteers' own plates, cups, glasses etc. The food items and dishes were entered into a computer and the results were converted into energy and nutrients using a computer program from the Swedish National Food Administration (Uppsala, Sweden). The mixed and vegetarian diets had previously been described and validated with biological markers [9,10].

\subsection{Clinical Examination and Chemical Analyses}

The clinical examination consisted of measurement of weight, height, heart rate, systolic and diastolic blood pressure. Subjects visited the clinic in the morning after a 12 hours fast. Body weight was measured (kg) on a digital scale in light clothing without shoes. Blood pressure was measured manually by cuff and stethoscope in a setting position on the right arm after a 5-min rest. Two measurements were performed with a 2-min interval, and the average value was calculated. All measurements were made by the same nurse throughout the study.

Blood samples were drawn from an antecubital vein using Vacutainer tubes. The samples were collected and handled according to hospital routines. Serum lipids were assayed by routine methods at the department of clinical chemistry, University hospital, Lund, Sweden. Control samples were run simultaneously.

\subsection{Statistical Analysis}

Results are presented as mean values with a 95\% confidence interval. Student's t-test for paired observations was used to compare mean values before (mixed diet) and 12 months after the dietary shift (lactovegetarian diet) as the aim was to study a long-term dietary change. The power calculation was made with the assumption of a decrease of HDL cholesterol of $0.14 \mathrm{mmol} / \mathrm{l}$, a standard deviation of 0.20 $\mathrm{mmol} / \mathrm{l}$, an alpha of 0.05 and a power of 0.80 . With these assumptions we would need at least 18 people to be able to detect a statistically significant effect of the intervention.

\section{RESULTS}

The major trends when changing from a mixed diet to a lactovegetarian diet were an increase in consumption of fruits, berries, vegetables, potatoes, dairy products, and herbal tea and a decrease in the intake of fish, egg, meat products, coffee and tea (Table 1). This led to an increase in

Table 1. Food intake (g/10 MJ) before (0 month) and 3, 6 and 12 months after the dietary shift. The results before the dietary shift are presented as mean values with $95 \%$ confidence intervals. The values for 3, 6 and 12 months after the dietary shift are the mean changes compared with the period before the dietary shift $(n=20)$.

\begin{tabular}{lcccc}
\hline & 0 month & 3 months & 6 months & 12 months \\
\hline Fruits, berries & $279 \pm 70$ & +188 & +135 & $+246^{* *}$ \\
Vegetables & $161 \pm 40$ & +200 & +193 & $+238^{* *}$ \\
Root vegetables & $147 \pm 41$ & +73 & +82 & $+100^{* *}$ \\
Potatoes & & & & \\
Meat, fish, egg & $161 \pm 34$ & -161 & -161 & $-159^{* *}$ \\
Milk, 0.5\% - 3\% fat & $461 \pm 95$ & +93 & +5 & +18 \\
Cheese & $39 \pm 10$ & +33 & +34 & $+32^{* *}$ \\
Cereals & $118 \pm 37$ & +77 & +48 & +43 \\
Bread, all kinds & $124 \pm 16$ & +42 & +45 & $+36^{* *}$ \\
Sweets & $5 \pm 5$ & -5 & -4 & $-5^{* *}$ \\
Alcoholic beverages & $62 \pm 41$ & -49 & -53 & $-60^{* *}$ \\
Coffee & $579 \pm 132$ & -564 & -574 & $-552^{* *}$ \\
Tea & $152 \pm 108$ & -143 & -138 & $-129^{* *}$ \\
Herbal tea & $12 \pm 14$ & +337 & +418 & $+680^{* *}$ \\
\hline
\end{tabular}

${ }^{* *} \mathrm{P}<0.01$ between 0 and 12 months. 
the intake of total carbohydrates and fibre and a decrease in fat, protein and sucrose (Table 2). There was no change in P/S-ratio (Table 1). The coronary heart disease risk markers body weight, body mass index (BMI), systolic and diastolic blood pressure, total cholesterol and low-density lipoprotein (LDL) cholesterol decreased significantly (Table 3).

\section{DISCUSSION}

The dietary survey was validated against biological markers for food intake; protein, sodium, potassium and fibre and also the Goldberg cut-off was calculated before, 3,6 and 12 months after the dietary shift [10]. The validation showed the same validity before and 3 months after the dietary shift and a declining validity towards 12 months with an underreporting of energy, protein, sodium and potassium but an overreporting of fibre, indicating a selective underreporting [10]. Nevertheless, the change in coronary heart disease risk markers was similar between 0 and 3 months and between 0 and 12 months (Table 3).

An unintentional weight loss of about two kg occurred during the one year duration of the study and a BMI loss of 0.6 units (Table 3). The weight and BMI loss was sustained during the one year period without a major dip initially, seen for some diets [11-13]. The main explanation of the change in coronary heart disease risk markers may thus be the weight loss effect of this diet.

One reason for the involuntary weight loss may be related to the physiological effects of the high fibre content of the vegetarian diet [14]. The impact of dietary fibres on satiation and satiety are related to the properties of adding bulk to the diet affecting gastric distension and produces viscosity in the gastric antrum and small intestine. Dietary fibre thus displaces available energy and nutrients from the diet, increase the time and work required for chewing and reduces the absorption efficiency of the small intestine. Moreover, regression of the heart vessel lesions may occur. Two-year weight loss diets have shown to induce a

Table 2. Nutrient intake before (0 month) and 3, 6 and 12 months after the dietary shift. The results are presented as mean values with 95\% confidence intervals $(n=20)$.

\begin{tabular}{lcccc}
\hline & 0 month & 3 months & 6 months & 12 months \\
\hline Carbohydrates (E\%) & $50 \pm 2$ & $56 \pm 2$ & $56 \pm 3$ & $58 \pm 2^{* * *}$ \\
Fat (E\%) & $36 \pm 2$ & $32 \pm 3$ & $32 \pm 3$ & $31 \pm 2^{* *}$ \\
Protein (E\%) & $15 \pm 1$ & $13 \pm 1$ & $13 \pm 1$ & $13 \pm 1^{*}$ \\
Sucrose (E\%) & $7.5 \pm 2.1$ & $4.9 \pm 1.3$ & $4.7 \pm 1.3$ & $5.8 \pm 1.6^{* *}$ \\
Fiber (g/10 MJ) & $25 \pm 3$ & $42 \pm 4$ & $43 \pm 3$ & $37 \pm 4^{* *}$ \\
P/S ratio & & $0.30 \pm 0.03$ & $0.28 \pm 0.050 .29 \pm 0.04$ & $0.28 \pm 0.06$ \\
\hline
\end{tabular}

${ }^{*} \mathrm{P}<0.05$ between 0 and 12 months; ${ }^{* *} \mathrm{P}<0.01$ between 0 and 12 months; ${ }^{1}$ The ratio polyunsaturated fatty acids to saturated fatty acids.
Table 3. Coronary heart disease risk markers before (0 month) and 3, 6 and 12 months after the dietary shift. The results are presented as mean values with 95\% confidence intervals $(n=20)$.

\begin{tabular}{lcccc}
\hline & 0 month & 3 months & 6 months & 12 months \\
\hline Body weight (kg) & $67.2 \pm 5.2$ & $64.9 \pm 4.6$ & $64.4 \pm 4.3$ & $65.3 \pm 4.7^{* *}$ \\
$\begin{array}{l}\text { BMI }\left(\mathrm{kg} / \mathrm{m}^{2}\right) \\
1.1^{* *}\end{array}$ & $23.4 \pm 1.3$ & $22.7 \pm 1.1$ & $22.5 \pm 1.1$ & $22.8 \pm 1.1^{* *}$ \\
$\begin{array}{l}\text { Systolic blood } \\
\text { pressure (mm·Hg) }\end{array}$ & $114 \pm 3$ & $112 \pm 4$ & $111 \pm 3$ & $109 \pm 4^{*}$ \\
$\begin{array}{l}\text { Diastolic blood } \\
\text { pressure (mm·Hg) }\end{array}$ & $79 \pm 3$ & $62 \pm 5$ & $62 \pm 5$ & $61 \pm 5^{*}$ \\
$\begin{array}{l}\text { Heart rate } \\
\text { (beats/min) }\end{array}$ & $66 \pm 4$ & $62 \pm 5$ & $62 \pm 5$ & $61 \pm 5^{*}$ \\
$\begin{array}{l}\text { Total cholesterol } \\
(\mathrm{mmol} / \mathrm{l})\end{array}$ & $5.81 \pm 0.40$ & $5.28 \pm 0.44$ & $5.37 \pm 0.50$ & $5.24 \pm 0.43^{*}$ \\
$\begin{array}{l}\text { HDL cholesterol } \\
\text { (mmol/l) }\end{array}$ & $1.64 \pm 0.19$ & $1.53 \pm 0.15$ & $1.44 \pm 0.14$ & $1.50 \pm 0.20^{*}$ \\
$\begin{array}{l}\text { LDL cholesterol } \\
\text { (mmol/l) }\end{array}$ & $3.74 \pm 0.39$ & $3.40 \pm 0.37$ & $3.52 \pm 0.47$ & $3.26 \pm 0.35^{*}$ \\
$\begin{array}{l}\text { LDL/HDL ratio } \\
\text { LD, }\end{array}$ & $2.48 \pm 0.45$ & $2.32 \pm 0.36$ & $2.62 \pm 0.52$ & $2.40 \pm 0.53$
\end{tabular}

${ }^{* *} \mathrm{P}<0.01$ between 0 and 12 months; ${ }^{*} \mathrm{P}<0.05$ between 0 and 12 months; ${ }^{1}$ High-density lipoprotein cholesterol; ${ }^{2}$ Low-density lipoprotein cholesterol.

significant regression of measurable carotid vessel wall volume [15]. The effect appears to be mediated mainly by the weight loss-induced decline in blood pressure. This is an important effect being as the volunteers could without any declared problems lose weight and sustain the weight loss for at least one year. There was no recommendation to lose weight. These studies indicate that the weight loss per se is more important than the relative distribution of fat, carbohydrates and protein and that vegetarian diets, mainly plant-based diets and so-called high-carbohydratelow-fat-diets are more satisfying than the opposite. Longterm studies (4 - 9 years) on low fat diets demonstrate a weight loss [16,17] and seem to prevent diabetes [18]. Prolonged consumption of diets low in carbohydrates and high in protein is associated with an increase in total mortality [19]. Positive associations of this score were noted with respect to both cardiovascular and cancer mortality. Similar results were obtained in a Swedish study [20]. Green tea, by some called herbal tea, was in some studies related to a decrease in body weight, waist circumference, body fat mass, skin-fold thickness, and total subcutaneous fat areas [21]. The mechanisms accounting for weight loss with tea or tea components are unclear [21]. It has been suggested that some tea components may increase sympathetic nervous system activity and stimulate thermogenesis, which would increase calorie consumption [22].

Besides the effect of certain food components, such as protein, fat, carbohydrates and fibre, the botanical struc- 
ture of the food is important for satiety. Experiments on different structures of foods, such as intact kernels and different milling fractions of cereals, solid or puréed fruits and vegetables have yielded different effects on satiation, satiety and subsequent ad libitum food (energy) intake [23-26]. The foods eaten after the dietary change in this study was in favour for botanical structures of foods leading to improved satiety and thus enhancing the probability of a lower total food (and energy) consumption.

Vegetarian diets seem to be protective against coronary heart diseases [6]. One reason for this is effects of plantbased diets on plasma lipids [2,27]. Twenty-seven randomized controlled and observational trials were included in a review article [27]. Of the four types of plant-based diets considered, interventions testing a combination diet, a vegetarian or vegan diet combined with nuts, soy, and/or fibre, demonstrated the greatest effects, up to $35 \%$ plasma LDL cholesterol reduction, followed by vegan and ovolacto-vegetarian diets. Vegetarian diets, such as this one, are characterised by a high intake of dietary fibre, among other sources from cereals. Several health promoting bioactive components are being associated with dietary fibre in cereals, for instance antioxidants such as tocotrienols, tocopherols, alkylresorcinols, phenolic acids, choline, betaine, lignans and sterols [28]. Most of these components are present in the bran and germ of the kernels. There was an increase in the consumption of fruits, berries, vegetables and potatoes in this study, which means an increase in dietary fibre, antioxidants and some important nutrients. Furthermore these foods contain plant sterols known to have plasma LDL lowering effects [29]. The serum lipid lowering effect of the vegetarian diet may have been mediated by several cholesterol-lowering dietary components in combination (a so-called dietary portfolio) presented by Jenkins et al. in several of his group's “portfolio-diet studies" [30]. The increase in fruits and vegetables was about 500 grams per day, which indicates that the recommendations from Sweden and the rest of Scandinavia and from many other countries on fruits and vegetables of about 500 grams per day seem to be sound.

Another interesting result was that the $\mathrm{P} / \mathrm{S}$ ratio was unchanged. Many of the meat products were replaced by dairy products leading to an unchanged intake of saturated and unsaturated fatty acids, and probably also transfatty acids. The decrease in total fat intake may be one explanation for the results. This is in contrast to many other studies that emphasize the importance of decreasing saturated fatty acids and a relative increase in polyunsaturated fatty acids [31-33]. One recent study in particular demonstrated that under isocaloric conditions a change from dairy fat to canola oil led to a decrease in risk markers for coronary heart diseases [31]. Unfortunately there was a trend to eat "natural" foods during the time our study took place. Butter was considered to be "natural" and margarine not to be "natural". Butter was thus recommended for use on bread and in cooking rather than margarines and oils.

Other explanations for the change in risk variables are sought for in the differences in carbohydrate and protein intake. In general, vegetarian diets derive more than 50\% of energy from carbohydrates, mainly from fruits, vegetables, legumes and whole-grain breads and cereals [5]. These low-fat, high-carbohydrate diets are associated with lower BMIs than are high-fat, low-carbohydrate diets. Results from the EPIC-Oxford study demonstrated that, of all the components of the diet, dietary fibre contributed the most to the age-adjusted differences in BMI, equivalent to body-weight differences of 3.6 and $2.7 \mathrm{~kg}$ for men and women, respectively [4]. When energy intake is ad libitum, the consumption of an additional $14 \mathrm{~g} / \mathrm{d}$ of dietary fibre is associated with a $10 \%$ decrease in energy intake and a body weight loss of $1.9 \mathrm{~kg}$ over 3.8 months [34]. The effect seems to be mediated by means of fibre reducing energy density and influencing lean body weight through effects on satiety and insulin control. In several studies relationships were found between a low protein percentage of the diet and a low BMI [5]. One explanation to this association is that down-regulation of insulin and up-regulation of glucagon may occur in response to the relative amount of non-essential amino acids in the diet $[35,36]$. Being as animal proteins have shown to have greater efficacy for releasing insulin, and plant proteins have a greater effect on glucagon, a plant-based diet may exert a downward effect on body weight [5].

There was a decrease in serum cholesterol $(-10 \%, \mathrm{P}<$ $0.01)$ in the present study, LDL cholesterol $(-13 \%, \mathrm{P}<$ $0.05)$, HDL cholesterol $(-9 \%, \mathrm{P}<0.05)$ and LDL/HDL $(-3 \%, n s)$. A similar result was obtained in a randomized controlled trial of a healthy Nordic diet, $-16 \%,-21 \%$, $-5 \%$ and $-14 \%$, respectively [37]. Even though the direction of the change was similar in both studies one difference between the studies was that in the present study there was no statistical significant reduction in the LDL/HDL ratio (Table 3) as it was in the NORDIET study [37]. The explanation may be that there was a higher intake of saturated fatty acids in this study compared to the NORDIET study [37] supporting the importance of polyunsaturated fatty acids in the diet. In both studies there was an involuntary weight loss, an increase in carbohydrate (\% of energy) and fibre (g/day) and a decrease in fat intake (\% of energy). It is plausible that the involuntary weight loss in both studies was induced by the increase in fibre intake. Both studies support current dietary guidelines in Europe, including the Nordic countries, as well as recommendations from the American Heart Association [38-40]. Thus, even though it is not necessary to eat a Scandinavian vegetarian diet to comply with nutritional recommendations, it seems to be as good an option as eating a Mediterranean diet or a healthy Nordic diet [37]. 
In the present study there was a trend towards a decrease in sodium intake according to the biological marker sodium in urine, from $2938 \mathrm{mg}$ before the dietary shift to $2870 \mathrm{mg} 12$ months after the dietary shift [10]. The potassium intake according to the biological marker potassium in urine and faeces was $3256 \mathrm{mg}$ before the dietary shift and 4119 mg 12 months after the dietary shift [10]. This may have affected the blood pressure, as well as the decrease in alcohol intake (Table 3). The effect on blood pressure of the decrease in coffee and tea consumption and the concomitant increase in herbal tea consumption is unclear, especially as we do not have any information on the type of herbal tea that the volunteers consumed. Nevertheless, we know that coffee can increase blood pressure and some herbal teas can decrease blood pressure [41]. This could thus preferably be investigated in future studies.

In conclusion, the positive effects of plant-based diets on CHD prevention may be mediated via a reduction in body weight; increased intake of carbohydrates, especially fibre intake and a decrease in energy, fat and protein intake. Primary, the main finding in this study is that there was a weight loss without any focus on this. Instead, the focus was on eating a healthy diet. Secondly, there was a decrease in LDL cholesterol and other CHD risk markers even though the P/S ratio was unchanged. Thirdly, when discussing the effects of vegetarian diets it is important to consider the presence or absence of certain food items, for instance vegetables, fruits, meat, coffee, tea and alcoholic beverages. Lastly, it is interesting to see that there is an effect on CHD risk variables within the normal BMI range. Thus, even those who fall within normal BMI ranges may benefit from either reducing weight or changing to a vegetarian diet, at least from BMI 23.4 to 22.8 according to this study.

\section{ACKNOWLEDGEMENTS}

Funding for the study was provided through grants from The Swedish Board for Planning and Coordination of Research, The Swedish Medical Research Council, The Swedish Foundation for Scientific Research without Animal Experiments, The Ekhaga Foundation and Hälsokostrådet. We certify that we do not have any affiliation with or financial involvement with any organization or entity with a financial interest in or financial conflict with the subject matter or materials discussed in the manuscript. GJ and BK took part in the data collection. GJ, BK and PAÖ took part in the trial design, conduct, analysis, interpretations of the outcome measures and drafting of the manuscript.

\section{REFERENCES}

[1] Fraser, G. (2009) Vegetarian diets: What do we know of their effects on common chronic diseases? American Journal of Clinical Nutrition, 89, 1607S-1612S. doi:10.3945/ajen.2009.26736K
[2] Rao, V. and Al-Weshaly, A. (2008) Plant-based diets and control of lipids and coronary heart disease risk. Current Atherosclerosis Reports, 10, 478-485. doi:10.1007/s11883-008-0075-2

[3] Micha, R., Sarah, K., Wallace, S. and Mozaffarian, D. (2010) Red and processed meat consumption and risk of incident coronary heart disease, stroke, and diabetes mellitus: A systematic review and meta-analysis. Circulation, 121, 2271-2283. doi:10.1161/CIRCULATIONAHA.109.924977

[4] Appleby, P., Thorogood, M., Mann, J. and Key, T. (1998) Low body mass index in non-meat eaters: The possible roles of animal fat, dietary fiber and alcohol. International Journal of Obesity and Related Metabolic Disorders, 22, 454-460. doi:10.1038/sj.ijo.0800607

[5] Berkow, S. and Barnard, N. (2006) Vegetarian diets and weight status. Nutrition Reviews, 64, 175-188. doi:10.1111/j.1753-4887.2006.tb00200.x

[6] Key, T., Appleby, P. and Rosell, M. (2006) Health effects of vegetarian and vegan diets. Proceedings of the Nutrition Society, 65, 35-41. doi:10.1079/PNS2005481

[7] Johansson, G., Holmén, A., Persson, L., Högstedt, B., Wassén, C., Ottova, L. and Gustafsson, J.-Å. (1992) The effect of a shift from a mixed diet to a lacto-vegetarian diet on human urinary and fecal mutagenic activity. Carcinogenesis, 13, 153-157. doi:10.1093/carcin/13.2.153

[8] Johansson, G., Ottova, L. and Gustafsson, J.-Å. (1990) Shift from a mixed diet to a lactovegetarian diet: Influence on some cancer-associated intestinal bacterial enzyme activities. Nutrition and Cancer, 14, 239-246. doi:10.1080/01635589009514099

[9] Johansson, G., Callmer, E. and Gustafsson, J.-Å. (1992) Changing from a mixed diet to a Scandinavian vegetarian diet: Effects on nutrient intake, food choice, meal pattern and cooking methods. European Journal of Clinical $\mathrm{Nu}$ trition, 46, 707-716.

[10] Johansson, G., Callmer, E. and Gustafsson, J.-Å. (1992) Validity of repeated dietary measurements in a dietary intervention study. European Journal of Clinical Nutrition, 46, 717-728.

[11] Dansinger, M., Glaeson, J., Griffith, J., Selker, H. and Schaefer, E. (2005) Comparison of the Atkins, Ornish, weight watchers, and zone diets for weight loss and heart disease risk reduction. Journal of the American Medical Association, 293, 43-53. doi:10.1001/jama.293.1.43

[12] Sacks, F., Bray, G., Carey, V., Smith, S., Ryan, D., Anton, S. and Williamson, D. (2009) Comparison of weight-loss diets with different compositions of fat, protein, and carbohydrates. New England Journal of Medicine, 360, 859873. doi:10.1056/NEJMoa0804748

[13] Shai, I., Schwarsfuchs, D., Henkin, Y., Shahar, D., Witkow, S., Greenburg, I. and Stampfer, M. (2008) Weight loss with a low-carbohydrate, Mediterranean, or low-fat diet. New England Journal of Medicine, 359, 229-241. doi:10.1056/NEJMoa0708681

[14] Heaton, K. (1973) Food fibre as an obstacle to energy intake. Lancet, 2, 1418-1421. doi:10.1016/S0140-6736(73)92806-7 
[15] Shai, I., Spence, J., Schwarzfuchs, D., Henkin, Y., Parraga, G., Rudich, A. and Stampfer, M. (2010) Dietary Intervention to reverse carotid atherosclerosis. Circulation, 121, 1200-1208. doi:10.1161/CIRCULATIONAHA.109.879254

[16] Howard, B., Van Horn, L., Hsia, J., Manson, J., Stefanick, M., Wassertheil-Smoller, S., et al. (2006) Low-fat dietary pattern and risk of cardiovascular disease: The women's health initiative randomized controlled dietary modification trial. Journal of the American Medical Association, 295, 655-666. doi:10.1001/jama.295.6.655

[17] Lindström, J., Peltonen, M., Eriksson, J., Louheranta, A., Fogelholm, M., Uusitupa, M. and Tuomilehto, J. (2006) High-fibre, low-fat diet predicts long-term weight loss and decreased type 2 diabetes risk: The Finnish Diabetes Prevention Study. Diabetologia, 49, 912-920. doi:10.1007/s00125-006-0198-3

[18] Galgani, J., Uauy, R., Aguirre, C. and Diaz, E. (2008) Effect of the dietary fat quality on insulin sensitivity. British Journal of Nutrition, 100, 471-479. doi:10.1017/S0007114508894408

[19] Trichopolou, A., Psaltopoulou, T., Orfanos, P., Hsieh, C.-C. and Trichopoulos, D. (2007) Low-carbohydratehigh-protein diet and long-term survival in a general population cohort. European Journal of Clinical Nutrition, 61, 575-581.

[20] Lagiou, P., Sandin, S., Weiderpass, E., Lagiou, A., Mucci, L., Trichopolous, D. and Adami, H.-O. (2007) Low-carbohydrate-high protein diet and mortality in a cohort of Swedish women. Journal of Internal Medicine, 261, 366-374. doi:10.1111/j.1365-2796.2007.01774.x

[21] Deka, A. and Vita, J. (2011) Tea and cardiovascular disease. Pharmacological Research, 64, 136-145. doi:10.1016/j.phrs.2011.03.009

[22] Dulloo, A., Seydoux, J., Girardier, L. and Chantre, P. (2000) Green tea and thermogenesis: Interactions between catechin-polyphenols, caffeine and symphatetic activity. International Journal of Obesity Metabaloci Disorders, 24, 252-258. doi:10.1038/sj.ijo.0801101

[23] Bolton, R., Heaton, K. and Burroughs, L. (1981) The role of dietary fiber in satiety, glucose, and insulin-studies with fruit and fruit juices. American Journal of Clinical Nutrition, 34, 211-217.

[24] Flood-Obbagy, J. and Rolls, B. (2009) The effect of fruit in different forms on energy intake and satiety at a meal. Appetite, 52, 416-422. doi:10.1016/j.appet.2008.12.001

[25] Haber, G., Murphy, D., Heaton, K. and Burroughs, L. (1977) Depletion and disruption of dietary fiber-effects on satiety, plasma-glucose, and serum insulin. Lancet, 2, 679-682. doi:10.1016/S0140-6736(77)90494-9

[26] Moorhead, S., Welch, R., Barbara, M., Livingstone, E., Mccourt, M., Burns, A. and Dunne, A. (2006) The effects of the fibre content and physical structure of carrots on satiety and subsequent intakes when eaten as part of a mixed meal. British Journal of Nutrition, 96, 587-595. doi:10.1079/BJN20061790

[27] Ferdowsian, H. and Barnard, N. (2009) Effects of plant- based diets on plasma lipids. American Journal of Cardiology, 104, 947-956.

doi:10.1016/j.amjcard.2009.05.032

[28] Fardet, A. (2010) New hypotheses for the health-protective mechanisms of whole-grain cereals: What is beyond fibre? Nutrition Research Reviews, 23, 65-134. doi:10.1017/S0954422410000041

[29] Baumgartner, S., Mensink, R. and Plat, J. (2011) Plant sterols and stanols in the treatment of dyslipidemia: New insights into targets and mechanisms related to cardiovascular risk. Current Pharmaceutical Design, 17, 922932. doi:10.2174/138161211795428795

[30] Jenkins, D., Jones, P., Lamarche, B., Kendall, C., Faulkner, D., Cermakova, L. and Frolich, J. (2011) Effect of a dietary portfolio of cholesterol-lowering foods given at 2 levels of intensity of dietary advice on serum lipids in hyperlipidemia. A randomized controlled trial. Journal of the American Medical Association, 306, 831-839. doi:10.1001/jama.2011.1202

[31] Iggman, D., Gustafsson, I.-B., Berglund, L., Vessby, B., Marckmann, P. and Riserús, U. (2011) Replacing dairy fat with rapeseed oil causes rapid improvement of hyperlipedaemia: A randomized controlled study. Journal of Internal Medicine, 270, 356-364. doi:10.1111/j.1365-2796.2011.02383.x

[32] Sacks, F. and Katan, M. (2002) Randomized clinical trials on the effects of dietary fat and carbohydrate on plasma lipoproteins and cardiovascular disease. American Journal of Medicine, 113, 13S-24S. doi:10.1016/S0002-9343(01)00987-1

[33] Warensjö, E., Sundström, J., Vessby, B., Cederholm, T. and Riserus, U. (2008) Markers of dietary fat quality and fatty acid desaturation as predictors of total and cardiovascular mortality: A population-based prospective study. American Journal of Clinical Nutrition, 88, 203-209.

[34] Howarth, N., Saltzman, E. and Roberts, S. (2001) Dietary fiber and weight regulation. Nutrition Reviews, 59, 129139. doi:10.1111/j.1753-4887.2001.tb07001.x

[35] McCarty, M. (1999) Vegan proteins may reduce risk of cancer, obesity, and cardiovascular disease by promoting increased glucagon activity. Medical Hypothesis, 53, 459485. doi:10.1054/mehy.1999.0784

[36] McCarty, M. (2000) The origin of western obesity: A role for animal protein? Medical Hypothesis, 54, 488-494. doi:10.1054/mehy.1999.0882

[37] Adamsson, V., Reumark, A., Fredriksson, I.-B., Hammarström, E., Vessby, B., Johansson, G. and Risérus, U. (2011) Effects of a healthy Nordic diet on cardiovascular risk factors in hypercholesterolaemic subjects: A randomized controlled trial (NORDIET). Journal of Internal Medicine, 269, 150-159. doi:10.1111/j.1365-2796.2010.02290.x

[38] Becker, W., Lyhne, N., Pedersen, A., Aro, A., Fogelholm, M. and Thórsdottír, I. (2004) Nordic Nutrition Recommendations 2004. Integrating nutrition and physical activity. Nordic Council of Ministers, Copenhagen.

[39] Lichtenstein, A., Appel, L. and M, B. (2006) Diet and lifestyle recommendations revision 2006: A scientific 
statement from the American Heart Association Nutrition Committee. Circulation, 114, 82-96.

doi:10.1161/CIRCULATIONAHA.106.176158

[40] World Health Organization Technichal Report Series 2003; 916: i-viii, 1-149, back cover (2003). Diet, nutrition and the prevention of chronic diseases, 916.
[41] Zhang, Z., Hu, G., Caballero, B., Appel, L. and Chen, L. (2011) Habitual coffee consumption and risk of hypertension: A systematic review and meta-analysis of prospective observational studies. American Journal of Clinical Nutrition, 93, 1212-1219. doi:10.3945/ajcn.110.004044 